\title{
Genetic variants associated with response to lithium treatment in bipolar disorder: a genome-wide association study
}

\begin{abstract}
Liping Hou*, Urs Heilbronner*, Franziska Degenhardt*, Mazda Adli, Kazufumi Akiyama, Nirmala Akula, Raffaella Ardau, Bárbara Arias, Lena Backlund, Claudio E M Banzato, Antoni Benabarre, Susanne Bengesser, Abesh Kumar Bhattacharjee, Joanna M Biernacka, Armin Birner, Clara Brichant-Petitjean, Elise T Bui, Pablo Cervantes, Guo-Bo Chen, Hsi-Chung Chen, Caterina Chillotti, Sven Cichon, Scott R Clark, Francesc Colom, David A Cousins, Cristiana Cruceanu, Piotr M Czerski, Clarissa R Dantas, Alexandre Dayer, Bruno Étain, Peter Falkai, Andreas J Forstner, Louise Frisén, Janice M Fullerton, Sébastien Gard, Julie S Garnham, Fernando S Goes, Paul Grof, Oliver Gruber, Ryota Hashimoto, Joanna Hauser, Stefan Herms, Per Hoffmann, Andrea Hofmann, Stephane Jamain, Esther Jiménez, Jean-Pierre Kahn, Layla Kassem, Sarah Kittel-Schneider, Sebastian Kliwicki, Barbara König, Ichiro Kusumi, Nina Lackner, Gonzalo Laje, Mikael Landén, Catharina Lavebratt, Marion Leboyer, Susan G Leckband, Carlos A López Jaramillo, Glenda MacQueen, Mirko Manchia, Lina Martinsson, Manuel Mattheisen, Michael J McCarthy, Susan L McElroy, Marina Mitjans, Francis M Mondimore, Palmiero Monteleone, Caroline M Nievergelt, Markus M Nöthen, Urban Ösby, Norio Ozaki, Roy H Perlis, Andrea Pfennig, Daniela Reich-Erkelenz, Guy A Rouleau, Peter R Schofield, K Oliver Schubert, Barbara W Schweizer, Florian Seemüller, Giovanni Severino, Tatyana Shekhtman, Paul D Shilling, Kazutaka Shimoda, Christian Simhandl, Claire M Slaney, Jordan W Smoller, Alessio Squassina, Thomas Stamm, Pavla Stopkova, Sarah K Tighe, Alfonso Tortorella, Gustavo Turecki, Julia Volkert, Stephanie Witt, Adam Wright, L Trevor Young, Peter P Zandi, James B Potash, J Raymond DePaulo, Michael Baver, Eva Z Reininghaus, Tomas Novák, Jean-Michel Aubry, Mario Maj, Bernhard T Baune, Philip B Mitchell, Eduard Vieta, Mark A Frye, Janusz K Rybakowski, Po-Hsiu Kuo, Tadafumi Kato, Maria Grigoroiu-Serbanescu, Andreas Reif, Maria Del Zompo, Frank Bellivier, Martin Schalling, Naomi R Wray, John R Kelsoe*, Martin Alda*, Marcella Rietschel*, Francis J McMahon*, Thomas G Schulze*
\end{abstract}

\section{Summary}

Background Lithium is a first-line treatment in bipolar disorder, but individual response is variable. Previous studies have suggested that lithium response is a heritable trait. However, no genetic markers of treatment response have been reproducibly identified.

Methods Here, we report the results of a genome-wide association study of lithium response in 2563 patients collected by 22 participating sites from the International Consortium on Lithium Genetics (ConLiGen). Data from common single nucleotide polymorphisms (SNPs) were tested for association with categorical and continuous ratings of lithium response. Lithium response was measured using a well established scale (Alda scale). Genotyped SNPs were used to generate data at more than 6 million sites, using standard genomic imputation methods. Traits were regressed against genotype dosage. Results were combined across two batches by meta-analysis.

Findings A single locus of four linked SNPs on chromosome 21 met genome-wide significance criteria for association with lithium response $\left(\mathrm{rs} 79663003, \mathrm{p}=1 \cdot 37 \times 10^{-8} ; \mathrm{rs} 78015114, \mathrm{p}=1 \cdot 31 \times 10^{-8} ; \mathrm{rs} 74795342, \mathrm{p}=3 \cdot 31 \times 10^{-9}\right.$; and rs75222709, $\left.\mathrm{p}=3 \cdot 50 \times 10^{-9}\right)$. In an independent, prospective study of 73 patients treated with lithium monotherapy for a period of up to 2 years, carriers of the response-associated alleles had a significantly lower rate of relapse than carriers of the alternate alleles $(p=0 \cdot 03268$, hazard ratio $3 \cdot 8,95 \%$ CI $1 \cdot 1-13 \cdot 0)$.

Interpretation The response-associated region contains two genes for long, non-coding RNAs (lncRNAs), AL157359.3 and AL157359.4. LncRNAs are increasingly appreciated as important regulators of gene expression, particularly in the CNS. Confirmed biomarkers of lithium response would constitute an important step forward in the clinical management of bipolar disorder. Further studies are needed to establish the biological context and potential clinical utility of these findings.

Funding Deutsche Forschungsgemeinschaft, National Institute of Mental Health Intramural Research Program.

\section{Introduction}

Bipolar disorder is an often-devastating psychiatric illness characterised by disruptive mood swings, with intervals of partial or full recovery. Bipolar disorder types I and II affect at least $2 \%$ of the world's population; subthreshold forms affect another 2\%. ${ }^{1}$ Bipolar disorder consumes a substantial portion of mental health resources. Worldwide, the direct and indirect costs are large, with an estimated US\$151 billion spent in the USA alone in $2009 .^{2}$ Moreover, up to $15 \%$ of individuals with bipolar disorder die by suicide. ${ }^{3}$

Mood stabilisers are the first-line mode of medication treatment for bipolar disorder. ${ }^{4}$ Among these drugs, lithium stands out as a preventive agent for manic episodes, ${ }^{5}$ suicide attempts, and death by suicide. ${ }^{6}$ Consequently, lithium is still recommended as a first-line treatment for bipolar disorder, even though individual 

M M Nöthen MD); Department of Psychiatry and Psychotherapy, CharitéUniversitätsmedizin Berlin, Campus Charité Mitte, Berlin, Germany (M Adli MD, T Stamm MD); Fliedner Klinik Berlin, Center for Psychiatry, Psychotherapy and Psychosomatic Medicine, Berlin, Germany (M Adli); Department of Biological Psychiatry and Neuroscience, Dokkyo Medical University School of Medicine, Mibu, Japan (K Akiyama MD); Unit of Clinical Pharmacology, Hospital University Agency of Cagliari, Cagliari, Italy (R Ardau MD, C Chillotti MD,

M Del Zompo MD); Department of Biologia Animal, Unitat d'Antropologia (Dp Biología Animal), Facultat de Biologia and Institut de Biomedicina (IBUB), Universitat de Barcelona, CIBERSAM, Barcelona, Catalonia, Spain (B Arias PhD, M Mitjans PhD); Department of

Molecular Medicine and

Surgery (L Backlund MD,

L Frisén MD, C Lavebratt PhD, $M$ Schalling MD,

S G Leckband BS), Department of Clinical Neuroscience (L Frisén), Department of

Neurobiology, Care sciences, and Society (U Ösby MD),

Karolinska Institutet and Center for Molecular Medicine, Karolinska University Hospital, Stockholm, Sweden; Child and Adolescent Psychiatry Research

Center, Stockholm, Sweden

(L Frisén); Department of

Psychiatry, University of

Campinas (Unicamp), Campinas, Brazil

(CE M Banzato MD,

(R Dantas MD); Bipolar

Disorder Program, Institute of

Neuroscience, Hospital Clinic,

University of Barcelona,

IDIBAPS, CIBERSAM, Barcelona, Catalonia, Spain

(A Benabarre MD PhD,

$\mathrm{F}$ Colom $\mathrm{PhD}$, E Jiménez $\mathrm{PhD}$ E Vieta MD PhD);

Neurobiological Background and Anthropometrics in Bipolar Affective Disorder, Department of Psychiatry, Medical University of Graz, Graz, Austria (S Bengesser MD, A Birner MD, N Lackner MSc,

E Z Reininghaus MD); Veterans

Administration, San Diego Healthcare System, San Diego, CA, USA (T Shekhtman MSc,
Research in context

\section{Evidence before this study}

Lithium is a mainstay in the treatment of bipolar disorder, also known as manic-depressive illness, and might exert neuroprotective effects in neurodegenerative disorders. However, little is known about lithium's mechanism of action. Individual response in bipolar disorder varies from excellent to very poor, with about $30 \%$ of patients considered good responders. Many genetic association studies of lithium response have been done, but samples were small, and replicable findings have not emerged. To our knowledge, three genome-wide association studies (GWAS) of lithium response have been published to date, each implicating different loci.

\section{Added value of this study}

The international Consortium on Lithium Genetics has assembled the largest GWAS on lithium response in bipolar disorder to date, totalling more than 2500 individuals. We now present genome-wide significant evidence of association between lithium response and common genetic variants on

response is variable. Many patients show a robust improvement with lithium and a subset is highly responsive, ${ }^{7-9}$ with near-total resolution of symptoms. However, at least $30 \%$ of patients are only partially responsive, and more than $30 \%$ have no clinical response to lithium.

Evidence suggests that some of the variability in lithium response has a genetic basis, but sample sizes in such studies have been small. Good responders are more likely to have a family history of bipolar disorder than poor responders. ${ }^{10}$ Patients who stabilise on lithium tend to aggregate within families. ${ }^{11,12}$ A twin study reported better lithium prophylaxis in twins whose co-twin also had bipolar disorder. ${ }^{13}$

Genetic markers of lithium response could provide insight into the biological mechanism of lithium action and might be valuable for treatment planning. However, few pharmacogenetic studies of lithium have been published, and those have generally used small samples and variable definitions of response. Candidate gene studies have focused on genes purported to be involved in the therapeutic action of lithium, but replicable results have not emerged. ${ }^{14,15}$ Three genome-wide association studies (GWAS) of lithium response have been published. The first was from the Systematic Treatment Enhancement Program for Bipolar Disorder (STEP-BD) cohort, ${ }^{16}$ in which 458 patients with bipolar disorder I/II were treated with lithium and response was evaluated as time to recurrence during lithium treatment. No genomewide significant results were identified. A second GWAS was done in 204 Sardinian patients with bipolar disorder (only 52 were genotyped with single nucleotide polymorphism [SNP] arrays). No SNPs reached genomewide significance. Most recently, Chen and colleagues ${ }^{18}$ chromosome 21. The genetic region associated with response contains two long non-coding RNA genes, which are increasingly appreciated as important regulators of gene expression, particularly in the CNS. These findings suggest a novel potential mechanism of action for lithium. In an independent, prospectively followed clinical sample, the identified genetic markers also helped predict relapse during lithium treatment.

Implications of all the available evidence

Our findings suggest that a better understanding of drug mechanisms and response can be achieved through international cooperative efforts that leverage clinical expertise with large-scale genomics. The genetic markers identified here show predictive value in a prospective clinical sample, but further studies are needed to establish the potential clinical usefulness of these findings and their biological context. Confirmed biomarkers of lithium response would be an important advance in clinical management of bipolar disorder.

performed a GWAS on 294 highly treatment-adherent individuals of Asian ancestry selected from a larger set of about 2000 treated for bipolar disorder I with lithium monotherapy. The authors reported genome-wide significant association with a cluster of SNPs at 3p24.1. However, to date, all other reported studies have failed to replicate these findings in either Asian or Europeanancestry samples. ${ }^{19-21}$

To overcome the problems inherent in smaller sample sizes, we established the international Consortium on Lithium Genetics in 2008. ${ }^{22}$ Here, we report the results of an initial GWAS of lithium response in 2563 patients with bipolar disorder - by far the largest sample to dateusing phenotype and genotype data from 22 ConLiGen sites from four continents (Europe, America, Asia, and Australia; appendix).

\section{Methods \\ Study design and participants}

Over the timeframe of this study (phenotyping between 2008 and 2013), available samples were collected and genotyped in two distinct phases. We thus analysed the data as two distinct GWAS, referred to as GWAS 1 and GWAS 2; a detailed rationale and the analysis pipeline is provided in the appendix.

A Diagnostic and Statistical Manual of Mental Disorders (DSM) III or DSM-IV diagnosis of a bipolar spectrum disorder (appendix) was required, along with data on sex and total score on the Retrospective Criteria of Long-Term Treatment Response in Research Subjects with Bipolar Disorder (Alda scale ${ }^{12}$ ). We included all patients in whom response could be reliably evaluated; patients were required to have taken lithium for a minimum of 6 months with no additional mood 
stabiliser added. Comorbid medical or psychiatric disorders were not among the exclusion criteria. After this step, 1162 individuals were included in GWAS 1 and 1401 were included in GWAS 2.

Written informed consent was obtained from all participants. Ethical and regulatory approvals were obtained at each site that contributed anonymised data and DNA to the analysis.

\section{Phenotypes}

We used the Alda scale for the evaluation of long-term treatment response to lithium. This scale measures the change in illness episodes in the course of treatment with lithium. Briefly, the Alda scale quantifies symptom improvement in the course of treatment (A score, range $0-10$ ), which is then weighted against five criteria (B score) that assess confounding factors, each scored 0 , 1 , or 2 . The total score is then derived by subtracting the total B score from the A score. Negative scores are set to 0 by default so that the total score ranges from 0 to 10 .

ConLiGen previously conducted a multistage inter-rater reliability study ${ }^{23}$ aimed at finding the optimum way in which Alda subscale values can be combined for response evaluation. We evaluated two main phenotypes for lithium response: a dichotomous phenotype (good vs poor response to lithium), which has been successfully used in previous studies, ${ }^{9,13}$ and a continuous phenotype (range 0-10). We found the most reliable dichotomous phenotype to be that which designated all subjects with a total score of 7 or higher as "responders". The most reliable continuous phenotype was found to be one that used the A score but excluded all individuals with a total B score greater than 4.

Significant SNPs from the ConLiGen study were genotyped in an independent, longitudinally-assessed sample (appendix). After screening for eligibility and initial assessment, patients were started on lithium and entered the stabilisation phase. The goal in this phase was to stabilise patients within 3 months on lithium monotherapy. Following this, patients were observed for 1 month to assure stabilisation after discontinuation of other medications. Patients then entered the maintenance phase and were followed at 2-4-month intervals for 2 years.

\section{Genotyping, quality control, and imputation}

DNA was extracted from peripheral blood samples. Samples were genotyped at the National Institute of Mental Health (Bethesda, MD, USA), Life \& Brain Center at the University of Bonn (Bonn, Germany), or Broad Institute (Cambridge, MA, USA) using either Affymetrix or Illumina SNP arrays (appendix), according to the manufacturers' protocols.

Quality control and imputation were carried out in batches corresponding to distinct SNP arrays and ethnicities. Six batches of data were used in GWAS 1, including five of European ancestry (Affymetrix 6.0, Human610/660W, HumanOmniExpress,

\begin{tabular}{|c|c|c|}
\hline & GWAS 1 & GWAS 2 \\
\hline \multicolumn{3}{|l|}{ All individuals } \\
\hline Number & 1162 & 1401 \\
\hline Age at interview, years & $47 \cdot 80(13.99)$ & $46.84(13.83)$ \\
\hline \multicolumn{3}{|l|}{ Sex } \\
\hline Men & $473(41 \%)$ & $614(44 \%)$ \\
\hline Women & $689(59 \%)$ & $787(56 \%)$ \\
\hline Alda scale A score & $6.03(3.14)$ & $6 \cdot 35(2 \cdot 90)$ \\
\hline Alda scale total B score & $2 \cdot 11(1.63)$ & $2.86(1.68)$ \\
\hline Alda scale total score & $4.29(3.32)$ & $3.90(3.02)$ \\
\hline \multicolumn{3}{|c|}{ Dichotomous phenotype: good response (Alda scale total score $\geq 7$ ) } \\
\hline Number & 361 & 342 \\
\hline Age at interview, years & $51 \cdot 72(14 \cdot 27)$ & $48.92(14.80)$ \\
\hline \multicolumn{3}{|l|}{ Sex } \\
\hline Men & $158(44 \%)$ & $165(48 \%)$ \\
\hline Women & $203(56 \%)$ & $177(52 \%)$ \\
\hline Alda scale A score & $9 \cdot 21(0.82)$ & $9 \cdot 36(0 \cdot 77)$ \\
\hline Alda scale total B score & $0.88(0.84)$ & $1.38(0.96)$ \\
\hline Alda scale total score & $8 \cdot 33(1.10)$ & $7.99(1.01)$ \\
\hline \multicolumn{3}{|c|}{ Dichotomous phenotype: poor response (Alda scale total score $\leq 6$ ) } \\
\hline Number & 801 & 1059 \\
\hline Age at interview, years & $45 \cdot 86(13 \cdot 44)$ & $46 \cdot 17(13 \cdot 44)$ \\
\hline \multicolumn{3}{|l|}{ Sex } \\
\hline Men & $315(39 \%)$ & $449(42 \%)$ \\
\hline Women & $486(61 \%)$ & $610(58 \%)$ \\
\hline Alda scale A score & $4.60(2 \cdot 71)$ & $5 \cdot 38(2 \cdot 66)$ \\
\hline Alda scale total B score & $2 \cdot 66(1.59)$ & $3 \cdot 34(1 \cdot 58)$ \\
\hline Alda scale total score & $2 \cdot 47(2 \cdot 19)$ & $2.58(2.14)$ \\
\hline \multicolumn{3}{|c|}{$\begin{array}{l}\text { Continuous phenotype (Alda scale A score, with total B score }>4 \\
\text { excluded) }\end{array}$} \\
\hline Number & 1065 & 1168 \\
\hline Age at interview, years & $48.12(14.00)$ & $46.97(13.84)$ \\
\hline \multicolumn{3}{|l|}{ Sex } \\
\hline Men & $427(40 \%)$ & $510(44 \%)$ \\
\hline Women & $638(60 \%)$ & $658(56 \%)$ \\
\hline Alda scale A score & $6 \cdot 13(3 \cdot 13)$ & $6 \cdot 52(2 \cdot 87)$ \\
\hline Alda scale total B score & $1 \cdot 78(1 \cdot 26)$ & $2 \cdot 35(1 \cdot 16)$ \\
\hline Alda scale total score & $4.59(3.28)$ & $4.40(2 \cdot 94)$ \\
\hline
\end{tabular}

Data are $n, n(\%)$, or mean (SD). Alda scale refers to the Retrospective Criteria of Long-Term Treatment Response in Research Subjects with Bipolar Disorder.

Table 1: Phenotypic characteristics of individuals used for the analyses

HumanOmni1-Quad, HumanOmni2.5), and one of Japanese ancestry (HumanOmni2.5). Five batches of data were used in GWAS 2, including four European-ancestry datasets (Affymetrix 6.0, Human660W, HumanOmni1Quad, HumanOmniExpress), and one Taiwanese dataset (HumanOmniExpress) not overlapping with the sample studied by Chen and colleagues. ${ }^{18}$ Quality control parameters for retaining SNPs and subjects, including relatedness checking and population stratification analysis, are detailed in the appendix.

Genotype imputation was done with the prephasing and imputation strategy ${ }^{24}$ implemented by SHAPEIT2 ${ }^{25}$ and minimac. ${ }^{26}$ The full 1000 Genomes Project dataset
J R Kelsoe MD); Department of Psychiatry, University of California San Diego, San Diego, CA, USA

(A Kumar Bhattacharjee MD, CM Nievergelt PhD,

T Shekhtman, P D Shilling MD,

J R Kelsoe); Department of Health Sciences Research (J M Biernacka PhD) and Department of Psychiatry and Psychology (J M Biernacka M A Frye MD), Mayo Clinic, Rochester, MN, USA; INSERM UMR-S 1144-Université Paris Diderot Pôle de Psychiatrie, AP-HP, Groupe Hospitalier Lariboisière-F Widal, Paris, France (C Brichant-Petitjean MD, F Bellivier MD); The Neuromodulation Unit, McGil University Health Centre, Montreal, QC, Canada (PCervantes MD); The University of Queensland, Queensland Brain Institute, Brisbane, Queensland, QLD, Australia (G-B Chen PhD, N R Wray PhD); Department of Psychiatry \& Center of Sleep Disorders, National Taiwan University Hospital, Taipei, Taiwan (H-C Chen MD); Institute of Epidemiology and Preventive Medicine, Nationa Taiwan University, Taipei,

Taiwan (P-H Kuo PhD); Human Genomics Research Group, Department of Biomedicine, University Hospital Basel, Basel, Switzerland (S Cichon, S Herms, P Hoffmann); Discipline of Psychiatry, University of Adelaide, Adelaide, SA, Australia (S R Clark MBBS, KO Schubert MD, B T Baune MD); Campus for Ageing and Vitality, Newcastl University, Newcastle upon Tyne, UK (D A Cousins PhD); Douglas Mental Health University Institute, McGill University, Montreal, QC, Canada (C Cruceanu BSC, GTurecki MD); Psychiatric Genetic Unit, Poznan University of Medical Sciences, Poznan, Poland

(P M Czerski PhD, J Hauser MD); Department of Mental Health and Psychiatry, Mood Disorders Unit-Geneva University Hospitals, Geneva, Switzerland (A Dayer MD, J-M Aubry MD); Inserm U955, Psychiatrie Translationnelle, Créteil, France (B Étain MD, S Jamain PhD); Mental Illness Research Theme, Neuroscience Research Australia, Sydney, NSW, 


\footnotetext{
Australia (J M Fullerton PhD, PR Schofield PhD DSc); School of Medical Sciences, University of New South Wales, Sydney, NSW, Australia (J M Fullerton, PR Schofield); Pôle de Psychiatrie Générale Universitaire, Centre Hospitalier Charles Perrens, Bordeaux, France (S Gard MD); Department of Psychiatry, Dalhousie University, Halifax, Nova Scotia, NS, Canada (J S Garnham BN, CM Slaney RN, M Alda MD); Department of Psychiatry and Behavioral Sciences, Johns Hopkins University, Baltimore, MD, USA (F S Goes MD, FM Mondimore MD, BW Schweizer RN, J R DePaulo MD, T G Schulze);
} Mood Disorders Center of Ottawa, Ottawa, ON, Canada (P Grof MD); Molecular Research Center for Children's Mental Development, United Graduate School of Child Development, Osaka University, Osaka, Japan (R Hashimoto MD); Service de Psychiatrie et Psychologie Clinique, Centre Psychothérapique de Nancy-Laxou-Université de Lorraine, Nancy, France (J-P Kahn MD PhD); Department of Psychiatry, Psychosomatic Medicine and Psychotherapy, University Hospital Frankfurt, Frankfurt, Germany (S Kittel-Schneider MD, J Volkert PhD, A Reif MD); Department of Adult

Psychiatry, Poznan University of Medical Sciences, Poznan, Poland (S Kliwicki MD, J K Rybakowski MD); Department of Psychiatry and Psychotherapeutic Medicine, Landesklinikum Neunkirchen, Neunkirchen, Austria (B König MSC); Department of Psychiatry, Hokkaido University Graduate School of Medicine, Sapporo, Japan (I Kusumi MD); Institute of Neuroscience and Physiology, The Sahlgrenska Academy at the Gothenburg University, Gothenburg, Sweden (M Landén MD); Department of Medical Epidemiology and Biostatistics (M Landén) and Department of Clinical Neurosciences (L Martinsson MD), Karolinska Institutet, Stockholm, Sweden; Assistance Publique-Hôpitaux

de Paris, Hôpital Albert Chenevier-Henri Mondor, Pôle de Psychiatrie, Créteil, France (M Leboyer MD); Department

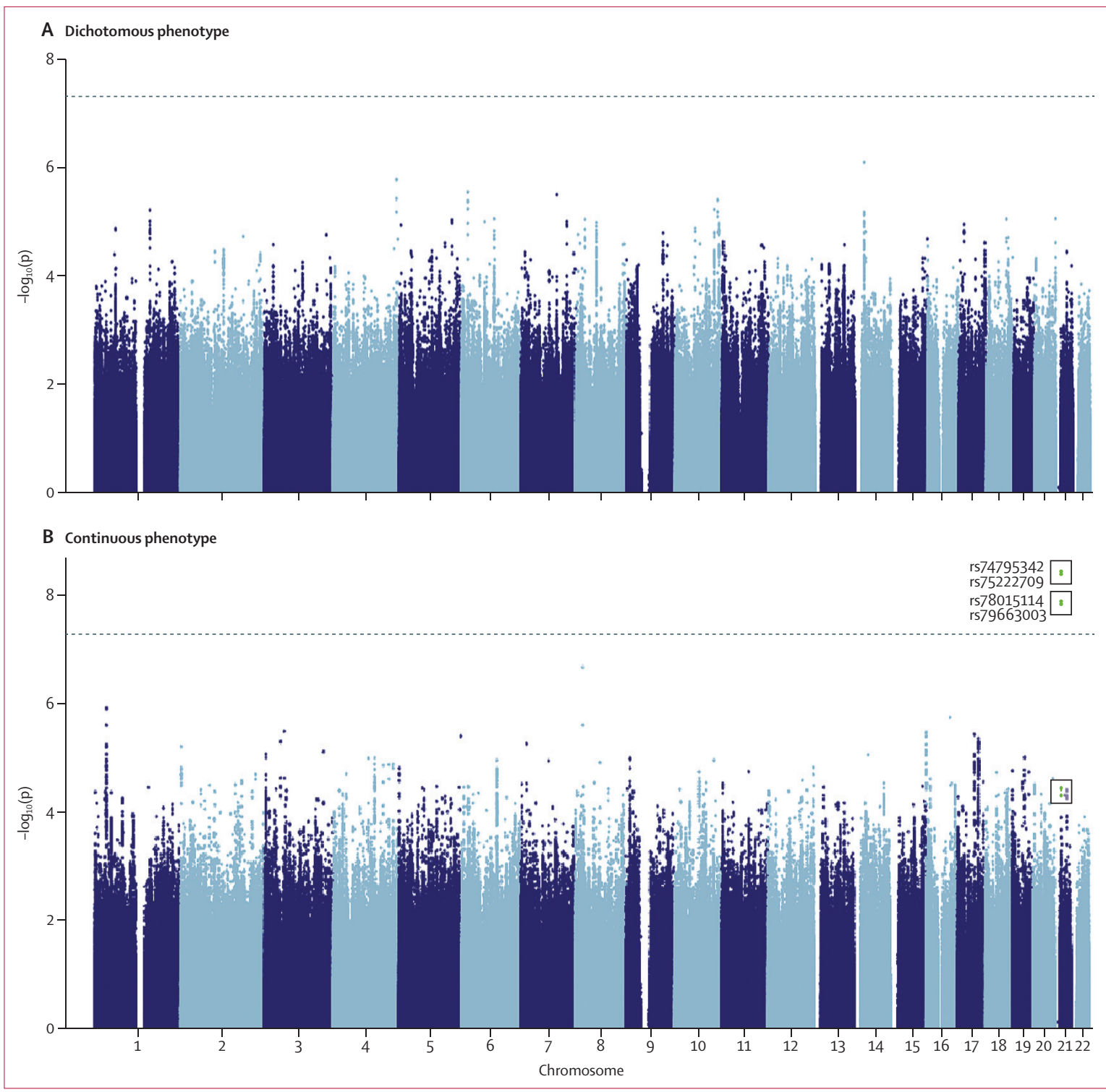

Figure 1: Meta-analysis results of dichotomous and continuous lithium response phenotypes in all participants

Genome-wide significant association at $\mathrm{p}<5 \times 10^{-8}$ (dotted line) can be detected with the continuous phenotype. SNPs in green (and outlined) are in linkage disequilibrium $\left(r^{2}>0 \cdot 6\right)$ with the index SNPs (rs74795342). SNP=single nucleotide polymorphism.

was used as the reference panel. Imputation was done separately for each SNP array and ancestry group. Gene dosages for all markers with imputation $r^{2} \geq 0.5$ in all batches were used for the final association tests.

\section{Statistical analysis}

We did association testing separately in Europeanancestry and Asian-ancestry samples. We analysed both the categorical and the quantitative response phenotypes. Using PLINK v1.07, ${ }^{27}$ we evaluated the association between allele dosages and the dichotomous phenotype by logistic regression, and the association between allele dosages and the quantitative phenotype was evaluated by linear regression. Genotyping platform was used as a covariate and, in the European-ancestry samples, the first four principal components of population structure were also included in the model to control for population stratification (appendix). Site of collection was not included as a covariate because it was highly colinear with genotyping platform. Results across GWAS 1 and GWAS 2 were combined by meta-analysis using METAL, ${ }^{28}$ under a fixed-effect model with heterogeneity testing.

Overall results in GWAS 1 were compared to those in GWAS 2 by use of the sign test (appendix). If there were no associations between SNPs and traits, the expectation is that $50 \%$ of the $\beta$ coefficients would have the same sign. The significance of the observed proportion was evaluated under the binomial distribution. 
To investigate the contribution of the bipolar disorder risk profile scores to lithium response, we used the linkage disequilibrium clumped complete result file of 108835 SNPs from the Psychiatric Genomics Consortium bipolar GWAS $^{29}$ to calculate $-\log$ (odds ratio [OR]) weighted risk profile score in each of the two Europeanancestry samples. Regression (using PLINK, v1.07) was then used to test whether the calculated risk profile scores had any effect on the association between SNP dosages and lithium response by adding the risk profile scores as an additional covariate in the regression model.

\section{Role of the funding source}

The funding bodies had no role in study design, data collection, data analysis, data interpretation, or writing of the report. LH, UH, FJM, and TGS had full access to all the data, except personal identifying information. The corresponding authors FJM and TGS had final responsibility for the decision to submit for publication.

\section{Results}

A total of 3193 participants were genotyped; 2563 remained after quality control (1162 in GWAS 1 and 1401 in GWAS 2). Study sites were largely nonoverlapping (appendix). Descriptive statistics of the phenotypes of the total sample analysed in the present study can be found in table 1; excluded participants are detailed in the appendix.

Our principal goal was to identify common genetic variants associated with differential response to lithium. Neither GWAS 1 nor GWAS 2 alone detected a genomewide significant result $\left(\mathrm{p}<5 \times 10^{-8}\right)$. However, there was greater-than-chance consistency between GWAS 1 and GWAS 2 in the overall direction of association. For the continuous phenotype, of 606 independent SNPs in GWAS 1 with $\mathrm{p}<0 \cdot 001,326$ (54\%) had the same sign in GWAS 2 . This represents a significantly greater agreement than chance alone $(p=0 \cdot 034)$. For the dichotomous phenotype, of 555 independent SNPs in GWAS 1 with $\mathrm{p}<0.001,317(57 \%)$ had the same sign in GWAS 2, significantly greater than chance $(\mathrm{p}=0 \cdot 0005)$. The complete list of SNPs used in this test is provided in the appendix.

When both studies were combined by meta-analysis, genome-wide significance was attained for the continuous phenotype (figures 1-3). Table 2 summarises the top results $\left(\mathrm{p}<1 \times 10^{-6}\right)$ with the continuous phenotype, and the appendix provides the top results with the dichotomous phenotype. A region on chromosome 21 contained four SNPs that showed genome-wide significant association with lithium response (minimum $\mathrm{p}=3 \cdot 31 \times 10^{-9}$; figure 2 ). These four SNPs are in very strong linkage disequilibrium with each other and have similar minor allele frequencies. The same four SNPs were associated with the dichotomous definition of lithium response at a $\mathrm{p}$ value of roughly $0 \cdot 01$. These four SNPs also reached significance when only the Europeanancestry population was considered (table 2). The imputation quality for these four SNPs was excellent and was supported by direct genotyping in a subset of the total sample (appendix).

The associated chromosomal region contains no known protein-coding genes. Two long, non-coding RNAs (lncRNAs) have been identified in the region, AL157359.4 (Ensembl version ENSG00000232193) and AL157359.3 (Ensembl version ENSG00000226204). Two of the SNPs (rs74795342 and rs75222709) are located in the intronic region of the gene, AL157359.3. The other

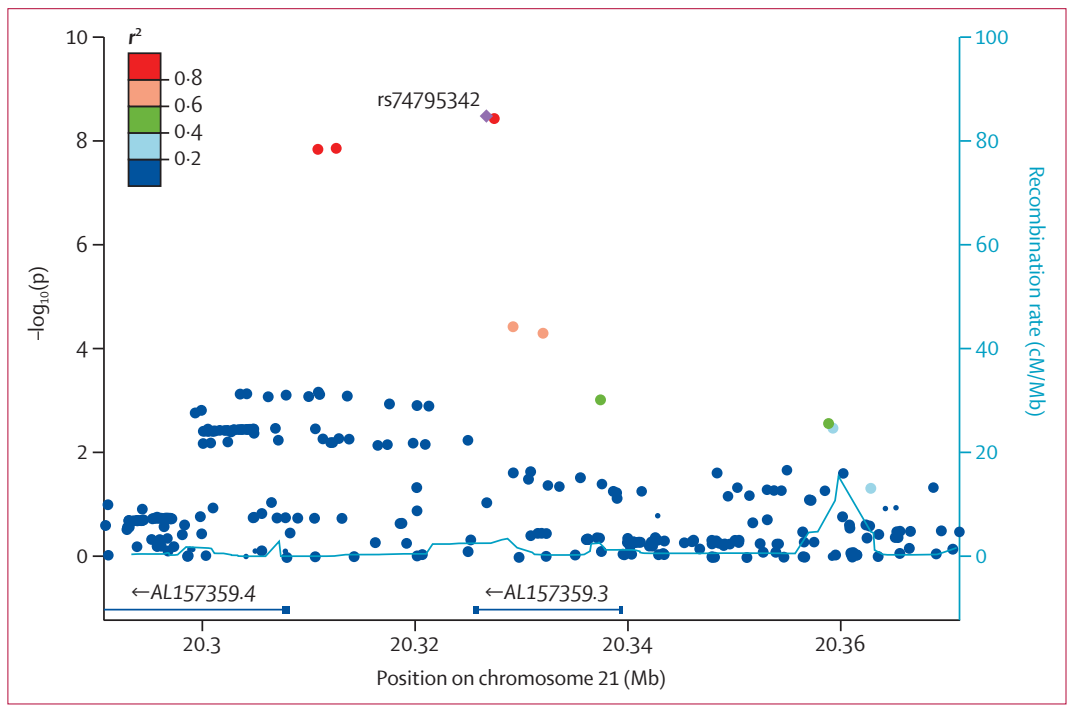

Figure 2: Regional association plot of the region on chromosome 21 in which the genome-wide significant SNPs are located

Association $\mathrm{p}$ values are plotted as points; colours indicate degree of linkage disequilibrium with index SNP (violet). Local recombination rate is shown as a solid blue line. Genes are indicated as straight blue lines labelled with gene names. $\mathrm{Mb}=$ megabase. $\mathrm{cM}=$ centimorgan. $\mathrm{SNP}=$ single nucleotide polymorphism.

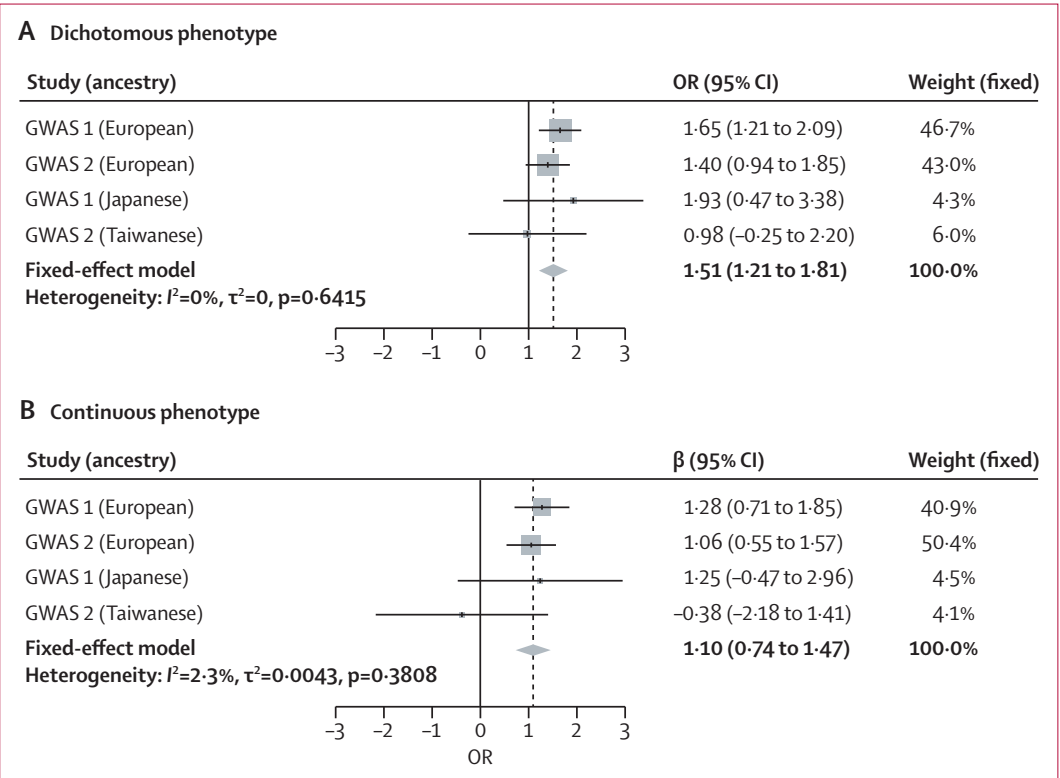

Figure 3: Forest plots for the most significant SNP, rs74795342

(A) Dichotomous phenotype. (B) Continuous phenotype. GWAS=genome-wide association study. OR=odds ratio. $\mathrm{SNP}=$ single nucleotide polymorphism. 


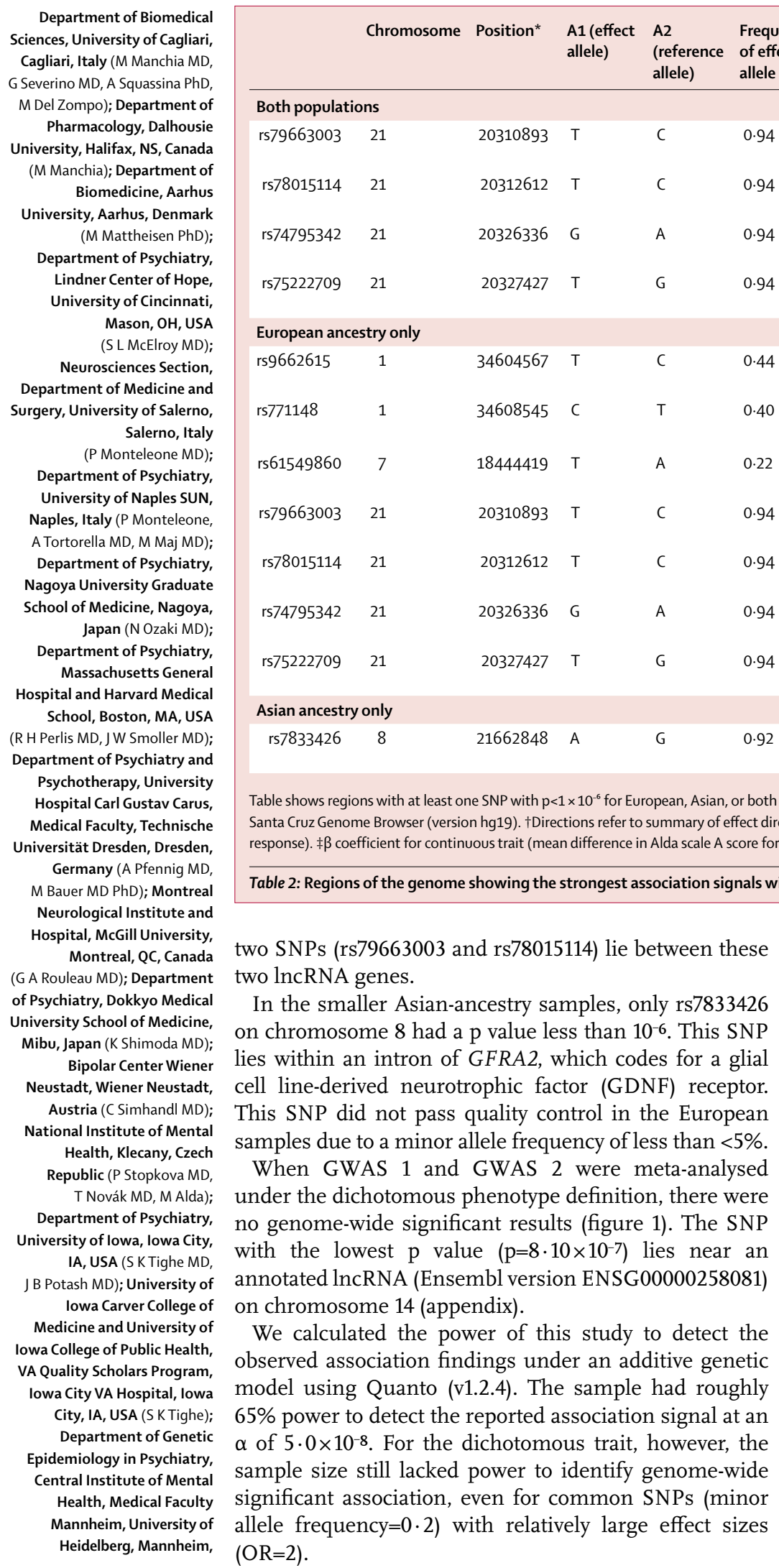

Department of Biomedical y, Aarhus, Denmark (M Mattheisen PhD); ment of Psychiatry, Mason, $\mathrm{OH}, \mathrm{USA}$ (S L McElroy MD); Department of Medicine and Salerno, Italy (P Monteleone MD); Department of Psychiatry, iversity of Naples SUN pital and Harvard Medica

ol, Boston, MA, USA Germany (A Pfennig MD

Bauer MD PhD); Montreal

Neurological Institute and ital, McGill University

(G A Rouleau MD); Department Psychiatry, Dokkyo Medical ustadt, Wiener Neustadt Health, Klecany, Czech ublic (P Stopkova MD ersity of lowa, lowa City, USA (S K Tighe MD, lowa Carver College of

Medicine and University of a College of Public Health City, IA, USA (S K Tighe); Department of Genetic idemiology in Psychiatry, institute of Mental Mannheim, University of

\begin{tabular}{|c|c|c|c|c|c|c|c|c|c|c|}
\hline & Chromosome & Position* & $\begin{array}{l}\text { A1 (effect } \\
\text { allele) }\end{array}$ & $\begin{array}{l}\text { A2 } \\
\text { (reference } \\
\text { allele) }\end{array}$ & $\begin{array}{l}\text { Frequency } \\
\text { of effect } \\
\text { allele }\end{array}$ & Gene & $p$ value & Directions $\dagger$ & $\beta(95 \% \mathrm{Cl}) \neq$ & $\begin{array}{l}\text { Heterogeneity } \\
\text { p } \$\end{array}$ \\
\hline \multicolumn{11}{|c|}{ Both populations } \\
\hline rs79663003 & 21 & 20310893 & $\mathrm{~T}$ & $\mathrm{C}$ & 0.94 & AL157359.4 & $1.37 \times 10^{-8}$ & +++- & $\begin{array}{l}1.04 \\
(0.68-1.40)\end{array}$ & $0 \cdot 30$ \\
\hline rs78015114 & 21 & 20312612 & $\mathrm{~T}$ & $C$ & 0.94 & AL157359.4 & $1.31 \times 10^{-8}$ & +++- & $\begin{array}{l}1.04 \\
(0.68-1.40)\end{array}$ & $0 \cdot 31$ \\
\hline rs74795342 & 21 & 20326336 & G & A & 0.94 & AL157359.3 & $3.31 \times 10^{-9}$ & +++- & $\begin{array}{l}1 \cdot 10 \\
(0 \cdot 74-1 \cdot 47)\end{array}$ & 0.46 \\
\hline rs75222709 & 21 & 20327427 & $\mathrm{~T}$ & G & 0.94 & AL157359.3 & $3.50 \times 10^{-9}$ & +++- & $\begin{array}{l}1 \cdot 10 \\
(0 \cdot 73-1 \cdot 46)\end{array}$ & 0.46 \\
\hline \multicolumn{11}{|c|}{ European ancestry only } \\
\hline rs9662615 & 1 & 34604567 & $\mathrm{~T}$ & $C$ & $0 \cdot 44$ & CSMD2 & $5.26 \times 10^{-7}$ & ++ & $\begin{array}{l}0.45 \\
(0.27-0.62)\end{array}$ & 0.83 \\
\hline rs771148 & 1 & 34608545 & C & $\mathrm{T}$ & $0 \cdot 40$ & CSMD2 & $7.01 \times 10^{-7}$ & ++ & $\begin{array}{l}0.45 \\
(0.27-0.63)\end{array}$ & 0.64 \\
\hline rs61549860 & 7 & 18444419 & $\mathrm{~T}$ & $A$ & $0 \cdot 22$ & HDAC9 & $5.44 \times 10^{-7}$ & ++ & $\begin{array}{l}0.59 \\
(0.36-0.83)\end{array}$ & 0.92 \\
\hline rs79663003 & 21 & 20310893 & $\mathrm{~T}$ & C & 0.94 & AL157359.4 & $1.30 \times 10^{-8}$ & ++ & $\begin{array}{l}1 \cdot 10 \\
(0 \cdot 72-1 \cdot 48)\end{array}$ & 0.41 \\
\hline rs78015114 & 21 & 20312612 & $\mathrm{~T}$ & $C$ & 0.94 & AL157359.4 & $1.25 \times 10^{-8}$ & ++ & $\begin{array}{l}1 \cdot 10 \\
(0 \cdot 72-1 \cdot 48)\end{array}$ & 0.41 \\
\hline rs74795342 & 21 & 20326336 & G & A & 0.94 & AL157359.3 & $3.00 \times 10^{-9}$ & ++ & $\begin{array}{l}1 \cdot 16 \\
(0 \cdot 78-1 \cdot 54)\end{array}$ & 0.63 \\
\hline rs75222709 & 21 & 20327427 & $\mathrm{~T}$ & G & 0.94 & AL157359.3 & $3.14 \times 10^{-9}$ & ++ & $\begin{array}{l}1 \cdot 16 \\
(0 \cdot 78-1 \cdot 54)\end{array}$ & 0.64 \\
\hline \multicolumn{11}{|c|}{ Asian ancestry only } \\
\hline rs7833426 & 8 & 21662848 & A & G & 0.92 & GFRA2 & $2.10 \times 10^{-7}$ & ++ & $\begin{array}{l}3 \cdot 66 \\
(2 \cdot 37-4 \cdot 94)\end{array}$ & 0.23 \\
\hline
\end{tabular}

Table shows regions with at least one SNP with $p<1 \times 10^{-6}$ for European, Asian, or both populations. $A=$ adenine. $C=$ cysteine. G=guanine. $T=$ thiamine. *University of $C$ alifornia Santa Cruz Genome Browser (version hg19). †Directions refer to summary of effect direction for each study (+ means individuals who carry the A1 allele have better lithium response). $\ddagger \beta$ coefficient for continuous trait (mean difference in Alda scale A score for each allele). Sp value for the meta-analysis heterogeneity test.

Table 2: Regions of the genome showing the strongest association signals with the continuous trait

two SNPs (rs79663003 and rs78015114) lie between these o lncRNA genes. lies within an intron of GFRA2, which codes for a glial cell line-derived neurotrophic factor (GDNF) receptor. This SNP did not pass quality control in the European When due to a minor allele frequency of less than $<5 \%$. under the dichotomous phenotype definition, there were with the annotated lncRNA (Ensembl version ENSG00000258081) on chromosome 14 (appendix). model using Quanto (v1.2.4). The sample had roughly $65 \%$ power to detect the reported association signal at an $\alpha$ of $5 \cdot 0 \times 10^{-8}$. For the dichotomous trait, however, the sample size still lacked power to identify genome-wide allele frequency $=0 \cdot 2$ ) with relatively large effect sizes $(\mathrm{OR}=2)$.
It is possible that lithium response is related to the overall genetic risk burden for bipolar disorder rather than to lithium per se. To assess this, we re-evaluated the association between the most significant SNPs in a model that corrected for differences in overall bipolar disorder risk burden (risk profile scores) in the European-ancestry samples. Similar results were obtained (appendix). The four SNPs on chromosome 21 continued to show genome-wide significant association with lithium response. There was also no detectable association between risk profile scores and Alda Score in this sample (data not shown). These results suggest that the findings are specific to lithium response and do not reflect genetic risk for bipolar disorder.

We assessed genetic association of lithium response in the subset of patients diagnosed with bipolar I disorder of our two GWAS datasets (GWAS 1 and GWAS 2). This narrower phenotype comprised about $79 \%(n=2020)$ of all participants. Results (appendix) showed robust association of the same four SNPs on chromosome 21 with the continuous lithium response trait, suggesting that these SNPs play a role in lithium response in individuals with more narrowly defined bipolar disorder. 
Retrospective assessment of lithium response, while reliable in previous studies and when assessed within ConLiGen, ${ }^{23}$ is limited by recall bias, incomplete information, and other sources of unmeasured variance. To evaluate the potential impact of these sources of error and test the identified SNPs in an independent sample, we genotyped all four SNPs in samples of patients with bipolar disorder who were treated with lithium monotherapy and assessed prospectively. The sample was recruited entirely from the San Diego Veterans Affairs Medical Center, USA. A total of 89 patients with bipolar disorder participated in the prospective study. Basic characteristics of this sample can be found in the appendix. After excluding 16 individuals due to screening failure, diagnosis change, voluntary withdrawal, and non-compliance, 73 patients with bipolar disorder (65 with type I, eight with type II) were used for the final data analyses.

After correction for several factors known to affect relapse (appendix), heterozygote carriers of the alleles associated with poorer lithium response showed a significantly higher rate of relapse than did carriers of the alternate alleles $(\mathrm{p}=0 \cdot 03268$, hazard ratio $3 \cdot 8$, 95\% CI 1.1-13.0; appendix).

\section{Discussion}

In this study, four linked SNPs met genome-wide significance criteria for association with a quantitative measure of lithium response. The associated locus has been annotated with two lncRNA genes. If replicated, these findings would constitute a novel genetic marker and could implicate lncRNAs in the mechanism of lithium response.

To our knowledge, this is the largest GWAS of lithium response in bipolar disorder published to date. In a sample of more than 2500 individuals, we detected genome-wide significant evidence of association with SNPs at a locus on chromosome 21. Further support for this finding was detected in a small, independent, prospectively ascertained sample of patients on lithium monotherapy. This finding could have important implications for our understanding of lithium's mechanism of action in bipolar disorder, although replication in independent samples is needed. Personal treatment planning on the basis of genetic data depends on identification of additional markers and their total contribution to differences between individuals in response to treatment. Detection of genome-wide significant markers for a phenotype is the first step in demonstrating if such a goal is achievable.

This study has several limitations. ConLiGen relies on retrospective ratings of treatment response, which lack precision and are subject to recall bias. However, response was rated using a well-validated instrument, ${ }^{12}$ previously shown to be reliable by members of the ConLiGen Consortium, ${ }^{23}$ and the results were supported in a prospectively assessed, independent sample. The ConLiGen sample encompassed a variety of patients from a range of ancestries and clinical settings. This is more representative of real-world clinical situations, in which patients present at various stages of bipolar disorder and with a range of illness severity, and underlines the robustness of our results. As for any GWAS of a complex trait, sample size is crucial. The ConLiGen sample size seems small when compared with GWAS of categorical disease phenotypes, for which sample sizes on the order of 10000 are often required. However, common alleles have been found to exert larger effects on pharmacogenomic traits, ${ }^{30,31}$ for which samples of 2500 cases are relatively large. On the other hand, the statistically significant excess agreement in the direction of association between GWAS 1 and GWAS 2 that we observed suggests that additional genome-wide significant associations might emerge from larger sample sizes.

Our results do not support previous reports of individual genes strongly associated with lithium response. ${ }^{16,18}$ Some of those reports were based on smaller samples that might not be comparable to those we studied. They could also represent false positives. Much larger sample sizes would be needed to exclude any particular genes in a GWAS, however.

Our main findings seem to implicate lncRNA genes. This implication is causally uncertain, because we have not yet linked allelic variation at the associated SNPs to expression or function of either transcript. There has, however, been an increasing appreciation of the role of lncRNAs in gene regulation, especially in the CNS. An ongoing study of gene expression in peripheral blood during and after acute episodes of bipolar disorder found apparently decreased expression of one of the lncRNAs identified within the association region (AL157359.3; $\mathrm{p}=0 \cdot 08$, fold change $=1 \cdot 17$ ) after an acute manic episode (Po-Hsiu Kuo, personal communication).

Even if confirmed, the clinical importance of these findings might be limited. The relatively low frequency of the response-associated alleles means that genetic testing would be uninformative in most patients. These and additional genetic markers from future studies could ultimately lead to a clinically informative test, ${ }^{32}$ but additional information from established predictors such as family history might be needed, as has been observed for other phenotypes. ${ }^{33}$ In line with similar approaches in the field, polygenic score analyses to predict lithium response could prove to be especially informative, provided that larger, adequately phenotyped samples become available. Clinical utility is a high bar, but the current dearth of good biomarkers of lithium response means that any robust genetic markers could constitute a real step forward.

Any GWAS is subject to experimental error. Type I error can occur, although stringent levels of genome-wide significance keep this to a minimum. Association findings might reflect unobserved variables. The alleles found to be associated with poor lithium response in this study could actually reflect something else, such as treatment adherence. Supportive results in a longitudinal,
Germany (SWitt PhD, $M$ Rietschel MD, T G Schulze); School of Psychiatry, University of New South Wales, and Black Dog Institute, Sydney, NSW Australia (A Wright MCP, P B Mitchell MBBS); Department of Psychiatry, University of Toronto, Toronto, ON, Canada (LTYoung MD); Department of Mental Health, Johns Hopkins Bloomberg School of Public Health, Baltimore, MD USA (P P Zandi PhD); Laboratory for Molecular Dynamics of Mental Disorders, RIKEN Brain Science Institute, Saitama, Japan (T Kato MD); and Biometric Psychiatric Genetics Research Unit, Alexandru Obregia Psychiatric Hospital, Bucharest, Romania (M Grigoroiu-Serbanescu PhD) Correspondence to: Thomas G Schulze, Institute of Psychiatric Phenomics and Genomics (IPPG), Medical Center of the University of Munich, Munich, Germany

thomas.schulze@med.unimuenchen.de or

Francis J McMahon, Human Genetics Branch and Genetic Basis of Mood and Anxiety Disorders Section National Institute of Mental Health Intramural Research Program, Bethesda, MD 20892-3719, USA mcmahonf@mail.nih.gov

For the Consortium on Lithium Genetics see

http://www.ConLiGen.org

See Online for appendix

For Quanto see http://biostats usc.edu/Quanto.html 
prospectively rated sample are encouraging, but because of distinct methods of rating lithium response these cannot be viewed as a replication of the ConLiGen results. However, relapse over the course of 2 years on lithium monotherapy is in some ways a better phenotype than that assessed by the Alda Scale, which relies on retrospective ratings. The fact that the same alleles were associated with both retrospective response and prospective relapse might actually increase the importance of the findings and their potential clinical relevance.

GWAS are best viewed as an important starting point for additional investigations. Before embarking on functional studies, future work will need to replicate and extend these findings using comparable ratings of lithium response in large samples. Because we could have missed some additional true positive markers due to power constraints, such studies should also target the longer list of SNPs that were associated with lithium response at less significant $\mathrm{p}$ values than formally reported here. Summary results for SNPs with $\mathrm{p}<5 \times 10^{-5}$ are posted at the ConLiGen website; the corresponding authors can be contacted for more complete summary results. Additional

For the Scientific Advisory Board see http://www.conligen. org/sab.html experimental work is needed to establish the functional SNP or SNPs and their biological effect, if any, in cellular or animal models. Such models could facilitate screening for other drugs that mimic lithium, thus generating novel therapeutic candidates suitable for further study.

\section{Contributors}

LH, UH, FD, JRK, MAl, MR, FJM, and TGS designed the study, contributed to analysis and interpretation of data, and wrote the first draft of the report. NA, H-CC, SC, AJF, MAF, SH, PH, SJ, MMat, MMN TSh, NRW, and PPZ provided further data analyses. LH did the statistical analyses and prepared the tables and figures. MAd, NA, MAl,

For the BipolLife network see http://www.bipolife.org
MB, SC, PMC, FD, MDZ, JH, UH, MLa, FJM, LH, RHP, EZR, MR, JKR, MS, PRS, TGS, PDS, JWS, and AS were responsible for study design. MAd, KA, MAl, RA, BA, J-MA, LB, CEMB, MB, BTB, FB, ABe, SB, AKB, ABi, CB-P, PC, SRC, FC, CCr, PMC, AD, MDZ, JRDP, BÉ, PF, LF, MAF, JMF, JSG, MG-S, PG, OG, RH, JH, SJ, EJ, J-PK, LK, TK, JRK, SK-S, SK, BK, P-HK, IK, NL, GL, MLa, MLe, SGL, GM, MMaj, MMan, LM, SLM, PBM, MMi, FMM, PM, TN, UÖ, NO, AP, JBP, DR-E, AR, EZR, MR, GAR, JKR, PRS, KOS, TGS, BWS, GS, PDS, KS, CS, CMS, TSt, PS, SKT AT, GT, EV, JV, SW, AW, LTY, and PPZ were responsible for patient recruitment. Patient in-depth phenotyping was carried out by MAd, KA, MAl, RA, BA, LB, MB, BTB, FB, ABe, FC, EJ, EV, SB, ABi, CB-P, ETB CCh, CCr, CRD, MDZ, JRDP, MAF, FSG, MG-S, PG, RH, LK, SK, P-HK, NL, MLa, and CL. All authors contributed to drafting the work or revising it critically for important intellectual content and made substantial contributions to the concept and design of the study and acquisition, analysis, and interpretation of data.

\section{Declaration of interests}

MAd has received a grant from Servier, speaker's fees from Servier, Lundbeck, Aristo, Parexel, Gilead, ViiV, Deutsche Bank, MSD, and MyTomorrows, plus a non-financial support from Lundbeck. KA has received speaker's fees from Taisho Toyama Pharmaceutical. MAl is funded by a grant of the Canadian Institutes of Health Research. MB has received speaker's fees from AstraZeneca, Pfizer, Lilly, Lundbeck, GlaxoSmithKline, Servier, and Ferrer Internacional. BÉ received non-financial support from Labex Biopsy and Fondation Fondamental. $\mathrm{RH}$ received grants and speaker honoraria from Dainippon Sumitomo Pharma and Novartis plus speaker honoraria from Eli Lilly Japan, GlaxoSmithKline, Hisamitsu Pharmaceutical, Janssen Pharmaceutical, Nippon Zoki Pharmaceutical, Otsuka Pharmaceutical, Astellas Pharma, Pfizer, and the Yoshitomiyakuhin Corporation. TK received a grant from Takeda Pharmaceutical and fees from Kyowa Hakko Kirin, Eli Lilly
Japan, Otsuka Pharmaceutical, GlaxoSmithKline, Taisho Toyama Pharmaceutical, Dainippon Sumitomo Pharma, Meiji Seika Pharma, Pfizer Japan, Mochida Pharmaceutical, Shionogi \& Co, Janssen Pharmaceutical, Yoshitomiyakuhin Corporation, Agilent Technologies, Astellas Pharma, and Wako Pure Chemical Industries. IK received grants and fees from Dainippon Sumitomo Pharma, Eisai, Eli Lilly, GlaxoSmithKline, Kyowa Hakko Kirin, Meiji Seika Pharma, MSD, Novartis, Otsuka, Ono Pharmaceutical, Pfizer, Tanabe Mitsubishi Pharma, Takeda Pharmaceutical, Shionogi, and Yoshitomi Pharmaceutical; he received grants from AbbVie GK, Asahi Kasei Pharma, Boehringer Ingelheim, Chugai Pharmaceutical, and Daiichi Sankyo and fees from Astellas Pharma and Janssen Pharmaceutical. MJM served as unpaid consultant for Pathway Genomic (San Diego, USA). SLM received a grant and fees from Naurex and Shire, further grants from Alkermes, Cephalon, Forest, Marriott Foundation, Orexigen Therapeutics, and Takeda Pharmaceutical, he further has served on the advisory boards for Bracket, Hoffmann-La Roche, MedAvante, Sunovion and received fees from Novo Nordisk. RHP received personal fees from RID Ventures, Genomind LLC, Healthrageous, Pfizer, Perfect Health, Proteus, and Psybrain. PRS received a grant from NHMRC. TGS received a grant and fees from Roche Pharmaceuticals. TSt received personal fees from Servier, Lundbeck, and Bristol-Myers Squibb. All above listed interests are outside of the submitted work. All other authors declare no competing interests.

\section{Acknowledgments}

We are greatly indebted to all the study participants without whom this research would not have been possible. We thank the members of our Scientific Advisory Board for critical input over the course of the project. This work was in part funded by the Deutsche Forschungsgemeinschaft (DFG; grant no RI 908/7-1; grant FOR2107, RI 908/11-1 to Marcella Rietschel, Michael Bauer, and Thomas G Schulze, NO 246/10-1 to MMN) and the Intramural Research Program of the National Institute of Mental Health (ZIA-MH00284311; NCT00001174). The genotyping was in part funded by the German Federal Ministry of Education and Research (BMBF) through the Integrated Network IntegraMent (Integrated Understanding of Causes and Mechanisms in Mental Disorders), under the auspices of the e:Med Programme (grants awarded to TGS, MR, and MMN). OG, AP, TSt, MB, AR, and TGS received support from the German Federal Ministry of Education and Research (BMBF) within the framework of the BipolLife network. MMN received support from the Alfried Krupp von Bohlen und Halbach-Stiftung. Franziska Degenhardt received support from the BONFOR Programme of the University of Bonn, Germany. EZR received funding from the Land Steiermark as principal investigator. MS received funds from the Swedish Research Council, Swedish Brain Foundation and funds from Karolinska Institutet and Karolinska University Hospital. Some data and biomaterials were collected as part of eleven projects (Study 40) that participated in the National Institute of Mental Health (NIMH) Bipolar Disorder Genetics Initiative. From 2003-07, the principal investigators and co-investigators were: Indiana University, Indianapolis, IN, R01 MH59545

(John Nurnberger, Marvin J Miller, Elizabeth S Bowman, N Leela Rau, P Ryan Moe, Nalini Samavedy, Rif El-Mallakh [University of Louisville], Husseini Manji [Johnson and Johnson], Debra A Glitz [Wayne State University], Eric T Meyer [Oxford University, UK], Carrie Smiley, Tatiana Foroud, Leah Flury, Danielle M Dick [Virginia Commonwealth University], Howard Edenberg); Washington University, St Louis, MO, R01 MH059534 (John Rice, Theodore Reich, Allison Goate, Laura Bierut [K02 DA21237]); Johns Hopkins University, Baltimore, R01 MH59533 (Melvin McInnis, J Raymond DePaulo Jr, Dean F MacKinnon, Francis M Mondimore, James B Potash, Peter P Zandi, Dimitrios Avramopoulos, Jennifer Payne); University of Pennsylvania, PA, R01 MH59553 (Wade Berrettini); University of California at San Francisco, CA, R01 MH60068 (William Byerley, Sophia Vinogradov); University of Iowa, IA, R01 MH059548 (William Coryell, Raymond Crowe); University of Chicago, IL, R01 MH59535 (Elliot Gershon, Judith Badner, Francis McMahon, Chunyu Liu, Alan Sanders, Maria Caserta, Steven Dinwiddie, Tu Nguyen, Donna Harakal); University of California at San Diego, CA, R01 MH59567 (John Kelsoe, Rebecca McKinney); Rush University, IL, R01 MH059556 (William Scheftner, Howard M Kravitz, Diana Marta, Annette Vaughn-Brown, Laurie Bederow); and NIMH 
Intramural Research Program, Bethesda, 1Z01MH002810-01 (Francis J McMahon, Layla Kassem, PsyD, Sevilla Detera-Wadleigh, Lisa Austin, Dennis L Murphy [Howard University], William B Lawson, Evarista Nwulia, Maria Hipolito). This work was supported by the NIH grants P50CA89392 from the National Cancer Institute and 5K02DA021237 from the National Institute of Drug Abuse. The Canadian part of the study was supported by a grant \#64410 from the Canadian Institutes of Health Research to MAl. We wish to thank Joanne Petite and Giselle Kraus for assistance with data collection. Collection and phenotyping of the Australian UNSW sample, by PBM, PRS, JMF, and AW, was funded by an Australian NHMRC Program Grant (No. 1037196). The collection of the Barcelona sample was supported by the Centro de Investigación en Red de Salud Mental (CIBERSAM) IDIBAPS (grant numbers PI080247, PI1200906, PI12/00018), and Secretaria d'Universitats i Recerca del Departament d'Economia i Coneixement (2014SGR1636 and 2014SGR398). J-MA and AD were supported by the Swiss National Science Foundation (grant number 32003B_125469 and NCCR Synapsy). DC was supported by a Medical Research Council Clinician Scientist Fellowship Award (MR/L006642/1). LF was supported by the Swedish Research Council (grant no 523-2011-3807). MG-S was supported by UEFISCDI, Romania, grant no 89/2012. P-HK was funded by the Taiwan Ministry of Science and Technology (grant no MST 99-2314-B-002-140-MY3 and 102-2314-B-002-117-MY3). CALJ was funded by the "Estrategia de Sostenibilidad 2014-2015" program of the University of Antioquia. TN was supported by the Ministry of Health of the Czech Republic (grant no IGA NT13891). JBP was supported by the Reuben Stoltzfus Bipolar Research Fund and with SKT received funding from the James Wah Fund and Project MATCH. TGS and UH received support from the Dr-Lisa-OehlerFoundation (Kassel, Germany). AS has a postdoctoral fellowship funded by the Sardinia Regional Government POR Sardegna FSE Operational Program of the Autonomous Region of Sardinia, European Social Fund 2007-2013-Axis IV Human Resources, Objective 1.3, Line of Activity 1.3.1. NRW was funded by Australian NHMRC Fellowships 613602 and 1078901. MMan is now a resident in the psychiatry training program at the Section of Psychiatry, Department of Public Health, Clinical and Molecular Medicine, University of Cagliari, Cagliari, Italy. This study used the high-performance computational capabilities of the Biowulf Linux cluster at the National Institutes of Health, Bethesda, MD. Genotyping for part of the Swedish sample was funded by the Stanley Center for Psychiatric Research at the Broad Institute.

\section{References}

1 Merikangas KR, Akiskal HS, Angst J, et al. Lifetime and 12-month prevalence of bipolar spectrum disorder in the National Comorbidity Survey replication. Arch Gen Psychiatry 2007; 64: 543-52.

2 Dilsaver SC. An estimate of the minimum economic burden of bipolar I and II disorders in the United States: 2009. J Affect Dis 2011; 129: 79-83.

3 Goodwin FK, Jamison KR. Manic-depressive illness: bipolar disorders and recurrent depression, 2nd edn. New York, NY: Oxford University Press, 2007.

4 Geddes JR, Miklowitz DJ. Treatment of bipolar disorder. Lancet 2013; 381: 1672-82.

5 Bauer MS, Mitchner L. What is a "mood stabilizer"? An evidence-based response. Am J Psychiatry2004; 161: 3-18.

6 Tondo L, Hennen J, Baldessarini RJ. Lower suicide risk with long-term lithium treatment in major affective illness: a meta-analysis. Acta Psychiatr Scand 2001; 104: 163-72.

7 Baldessarini RJ, Tondo L. Does lithium treatment still work? Evidence of stable responses over three decades. Arch Gen Psychiatry 2000; 57: 187-90.

8 Rybakowski JK, Chlopocka-Wozniak M, Suwalska A. The prophylactic effect of long-term lithium administration in bipolar patients entering treatment in the 1970s and 1980s. Bipolar Dis 2001; 3: 63-67.

9 Garnham J, Munro A, Slaney C, et al. Prophylactic treatment response in bipolar disorder: results of a naturalistic observation study. J Affect Dis 2007; 104: 185-90.

10 Kleindienst N, Engel R, Greil W. Which clinical factors predict response to prophylactic lithium? A systematic review for bipolar disorders. Bipolar Dis 2005; 7: 404-17.
11 Duffy A, Alda M, Milin R, Grof P. A consecutive series of treated affected offspring of parents with bipolar disorder: is response associated with the clinical profile? Can J Psychiatry 2007; 52: 369-76.

12 Grof P, Duffy A, Cavazzoni P, et al. Is response to prophylactic lithium a familial trait? J Clin Psychiatry 2002; 6: 942-47.

13 Mendlewicz J, Verbanck P, Linkowski P, Wilmotte J. Lithium accumulation in erythrocytes of manic-depressive patients: an in vivo twin study. Br J Psychiatry1978; 133: 436-44.

14 McCarthy MJ, Leckband SG, Kelsoe JR. Pharmacogenetics of lithium response in bipolar disorder. Pharmacogenomics 2010; 11: 1439-65.

15 Can A, Schulze TG, Gould TD. Molecular actions and clinical pharmacogenetics of lithium therapy. Pharmacol Biochem Behav 2014; 123: 3-16.

16 Perlis RH, Smoller JW, Ferreira MA, et al. A genomewide association study of response to lithium for prevention of recurrence in bipolar disorder. Am J Psychiatry2009; 166: 718-25.

17 Squassina A, Manchia M, Borg J, et al. Evidence for association of an ACCN1 gene variant with response to lithium treatment in Sardinian patients with bipolar disorder. Pharmacogenomics 2011; 12: $1559-69$.

18 Chen CH, Lee CS, Lee MT, et al. Variant GADL1 and response to lithium therapy in bipolar I disorder. N Engl J Med 2014; 370: $119-28$.

19 Consortium on Lithium Genetics. Variant GADL1 and response to lithium in bipolar I disorder. N Engl J Med 2014; 370: 1857-59.

20 Ikeda M, Kondo K, Iwata N. Variant GADL1 and response to lithium in bipolar I disorder. N Engl J Med 2014; 370: 1856-57.

21 Cruceanu C, Alda M, Dion PA, Turecki G, Rouleau GA. No evidence for GADL1 variation as a bipolar disorder susceptibility factor in a Caucasian lithium-responsive cohort. Am J Psychiatry 2015; 172: 94-95

22 Schulze TG, Alda M, Adli M, et al. The International Consortium on Lithium Genetics (ConLiGen): an initiative by the NIMH and IGSLI to study the genetic basis of response to lithium treatment. Neuropsychobiology 2010; 62: 72-78.

23 Manchia M, Adli M, Akula N, et al. Assessment of response to lithium maintenance treatment in bipolar disorder: a Consortium on Lithium Genetics (ConLiGen) report. PLoS One 2013; 8: e65636.

24 Howie B, Fuchsberger C, Stephens M, Marchini J, Abecasis GR. Fast and accurate genotype imputation in genome-wide association studies through pre-phasing. Nat Genet 2012; 44: 955-59.

25 Delaneau O, Zagury JF, Marchini J. Improved whole-chromosome phasing for disease and population genetic studies. Nat Methods 2013; 10: 5-6.

26 Fuchsberger C, Abecasis GR, Hinds DA. minimac2: faster genotype imputation. Bioinformatics 2015; 31: 782-84.

27 Purcell S, Neale B, Todd-Brown K, et al. PLINK: a tool set for whole-genome association and population-based linkage analyses. Am J Hum Genet 2007; 81: 559-75.

28 Willer CJ, Li Y, Abecasis GR. METAL: fast and efficient meta-analysis of genomewide association scans. Bioinformatics 2010; 26: 2190-91.

29 Psychiatric GWAS Consortium Bipolar Disorder Working Group. Large-scale genome-wide association analysis of bipolar disorder identifies a new susceptibility locus near ODZ4. Nat Genet 2011 43: 977-83.

30 Aberg K, Adkins DE, Bukszar J, et al. Genomewide association study of movement-related adverse antipsychotic effects. Biol Psychiatry 2010; 67: 279-82.

31 Malhotra AK, Correll CU, Chowdhury NI, et al. Association between common variants near the melanocortin 4 receptor gene and severe antipsychotic drug-induced weight gain Arch Gen Psychiatry 2012; 69: 904-12.

32 Grande I, Berk M, Birmaher B, Vieta E. Bipolar disorder. Lancet 2015; published online Sept 17. http://dx.doi.org/10.1016/s01406736(15)00241-X.

33 Do CB, Hinds DA, Francke U, Eriksson N. Comparison of family history and SNPs for predicting risk of complex disease. PLoS Genet 2012; 8: e1002973.
For the Biowulf Linux cluster see http://hpc.nih.gov 